\title{
Laser and cavity cooling of a mechanical resonator with a Nitrogen-Vacancy center in diamond
}

\author{
Luigi Giannelli, ${ }^{1}$ Ralf Betzholz, ${ }^{1}$ Laura Kreiner, ${ }^{2}$ Marc Bienert, ${ }^{1}$ and Giovanna Morigi ${ }^{1}$ \\ ${ }^{1}$ Theoretische Physik, Universität des Saarlandes, 66123 Saarbrücken, Germany \\ ${ }^{2}$ Experimentalphysik, Universität des Saarlandes, 66123 Saarbrücken, Germany
}

(Dated: March 1, 2022)

\begin{abstract}
We theoretically analyse the cooling dynamics of a high-Q mode of a mechanical resonator, when the structure is also an optical cavity and is coupled with a NV center. The NV center is driven by a laser and interacts with the cavity photon field and with the strain field of the mechanical oscillator, while radiation pressure couples mechanical resonator and cavity field. Starting from the full master equation we derive the rate equation for the mechanical resonator's motion, whose coefficients depend on the system parameters and on the noise sources. We then determine the cooling regime, the cooling rate, the asymptotic temperatures, and the spectrum of resonance fluorescence for experimentally relevant parameter regimes. For these parameters, we consider an electronic transition, whose linewidth allows one to perform sideband cooling, and show that the addition of an optical cavity in general does not improve the cooling efficiency. We further show that pure dephasing of the NV center's electronic transitions can lead to an improvement of the cooling efficiency.
\end{abstract}

\section{INTRODUCTION}

Colour centers in diamond are widely studied because of their exceptional properties as bright solid-state quantum emitters at room temperature [1, 2]. Their dynamics is being analysed in a wide variety of setups, which for instance can achieve the strong coupling with high-finesse optical resonators [3 5] and/or the strain coupling with high-Q vibrating structures 44-10] or standing mechanical waves [11, 12. This experimental progress makes NV centers promising candidates for realizing quantum hybrid devices, namely, devices capable of interfacing photons, phonons, and spin excitations in a controlled way, and can offer a wide range of applications for quantum information processing [13 16] and quantum sensing $13,17+20$. It thus calls for identifying the perspectives for control of these hybrid devices, which requires a systematic characterization of their dynamics.

In this work, we theoretically analyse laser cooling of a high-Q vibrating mode, which is strain coupled to the electronic transitions of an NV-center in diamond and optomechanically coupled to an optical cavity. This situation can be realised, for instance, when NV center, high$\mathrm{Q}$ mechanical mode, and optical resonators are assembled in a monolithic diamond structure, as illustrated in Fig.1 and recently discussed in Refs. [9, 10. In this setup the high-Q vibrating mode can be optomechanically cooled by the coupling with the cavity and/or laser-cooled by the strain-coupling with the NV-center transitions between the state $|g\rangle \equiv\left|{ }^{3} A_{20}\right\rangle$ and the levels $\left|E_{x}\right\rangle \equiv|x\rangle$ and $\left|E_{y}\right\rangle \equiv|y\rangle$, sketched in Fig. 1(b). The starting point of our study is the theoretical model of Ref. 21, where the authors investigated the effect of the NV multilevel structure on the dynamics of a high-Q vibrational mode. We extend this model by including the high-finesse mode of an optical cavity, which couples to the electronic transitions of the NV center and to the mechanical resonator by means of radiation pressure, and determine the laser cooling dynamics. We focus in particular on the regime where the linewidth of the resonances induced by the coupling with the cavity is of the same order as the one of the electronic transitions of the NV center. We further determine the effect of pure dephasing, which tends to destroy the coherence of the NV-center excitations, on the cooling dynamics. Surprisingly, we identify regimes where pure dephasing can improve the cooling rate.

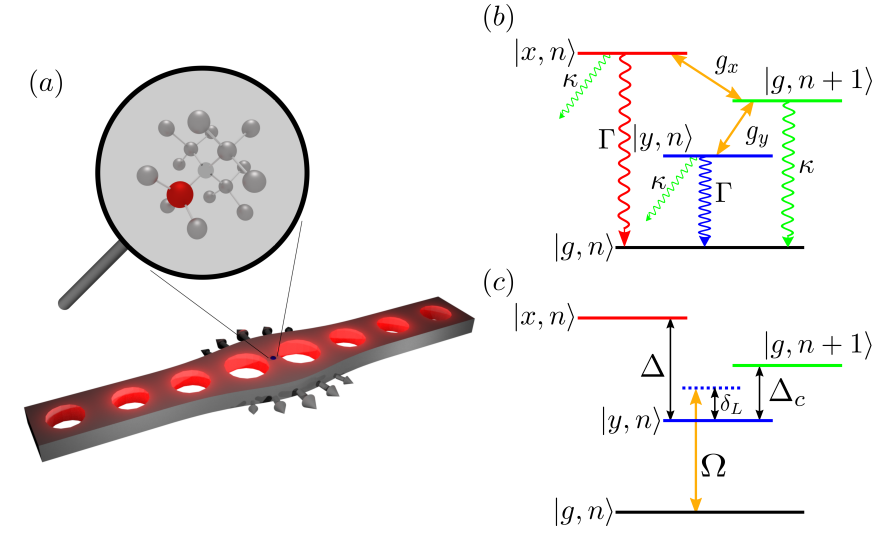

FIG. 1. (Color online) (a) A mechanical resonator, which is also a photonic crystal, interacts with a NV center in a diamond bulk via strain coupling. (b) The NV-center internal level structure, including the photonic excitations: the ground state $|g\rangle \equiv\left|{ }^{3} A_{20}\right\rangle$ couples to the excited states $|x\rangle \equiv\left|E_{x}\right\rangle$ and $|y\rangle \equiv\left|E_{y}\right\rangle$, which radiatively decay at rate $\Gamma$. A mode of the high-finesse optical cavity decays at rate $\kappa$ and drives quasiresonantly the transitions $|g, n+1\rangle \rightarrow|x, n\rangle,|y, n\rangle$ with $n$ the intracavity photon number. Coefficients $g_{x}$ and $g_{y}$ denote the corresponding vacuum Rabi frequencies. (c) Sketch of the relevant frequencies $\delta_{\mathrm{L}}, \Delta_{\mathrm{c}}, \Delta$, as a function of which the cooling efficiency is characterised, in presence of a laser driving the transition $|g\rangle \rightarrow|y\rangle$ with Rabi frequency $\Omega$.

This article is organised as follows. In Sec. II we review some general concepts ruling the cooling dynamics in presence of the strong coupling with a resonator. In 
Sec. III the theoretical model is introduced and in Sec. IV the parameter regime is discussed with reference to existing experimental realisation. In Sec. $\mid \mathrm{V}]$ the rate equations for the phonon dynamics are derived and in Sec. VI the cooling rate, the asymptotic temperature, and the spectrum of resonance fluorescence are determined and discussed in the presence and in the absence of the coupling with the optical cavity mode. Moreover, the cooling efficiency as a function of the dephasing rate is analysed. The conclusions are drawn in Sec. VII.

\section{GENERAL CONSIDERATIONS}

Our study is motivated by an experimentally existing platform, like the one sketched in Fig. 1. Our purpose is to investigate whether the optomechanical coupling can help in achieving lower temperatures than the ones that have been predicted by sideband cooling using the straincoupling with the NV center, see Ref. [21]. In fact, there can be an advantage by resorting to the optical cavity if the final occupation of the mechanical oscillator is lower than by just performing sideband cooling with the NV center, and thus if (i) the cavity-assisted cooling processes are sufficiently faster than the thermalization with the external environment and yet (ii) the final occupation of the oscillator is smaller than the one obtained by solely employing sideband cooling, according to a protocol like the one described in Ref. [21]. This analysis draws from several works where it was studied how the interplay between the mechanical effects due to the coupling of an electronic transition with a laser and with a cavity can increase the cooling efficiency of a mechanical oscillator 22 25]. There it was found that ground state cooling can be achieved as long as the mechanical oscillator frequency, here denoted by $\nu$, is larger than either the linewidth of the electronic transition, $\Gamma$, or of the optical resonator, $\kappa$. The minimal final mechanical oscillator occupation one can achieve is then controlled by the ratio between the linewidth of the narrower resonance, which we denote here by $\Gamma_{\min }=\min (\kappa, \Gamma)$, and $\nu$. Accordingly, the cooling rate $\tilde{\Gamma}$ is slower and scales with $\Gamma_{\min }$.

These dynamics can be often illustrated by means of a set of rate equations for the occupations $p_{n}$ of the oscillator's state with $n$ excitations $(n=0,1,2, \ldots)$ [26]:

$$
\dot{p}_{n}=-n\left(A_{+}+A_{-}\right) p_{n}+(n+1) A_{-} p_{n+1}+n A_{+} p_{n-1},
$$

with $\sum_{n} p_{n}=1$ (see Sec. $\mathrm{V}$ for details how this equation is derived). Here, $n A_{+}$and $n A_{-}$are the rates with which the oscillator in state $|n\rangle$ is heated and cooled, respectively, by one phonon, and can have the form of lorentz functions, whose linewidth is determined by the linewidth scattering resonance.

Specific predictions for the relevant quantities, whose dynamics Eq. (1) describes, can be directly extracted from the equation for the mean phonon occupation num- $\operatorname{ber}\langle n\rangle=\left\langle a^{\dagger} a\right\rangle=\sum_{n} n p_{n}[26]$ :

$$
\langle\dot{n}\rangle=-(\tilde{\Gamma}+\gamma)\left(\langle n\rangle-n_{f}\right)
$$

Here $\gamma$ is the thermalization rate and $n_{f}$ the final phonon occupation of the mechanical mode. Finally

$$
\tilde{\Gamma}=A_{-}-A_{+}
$$

is the cooling rate when $A_{-}>A_{+}$, whose maximum amplitude scales as $\tilde{\Gamma} \sim\left(\omega_{r} / \nu\right) \Gamma_{\min }$ with $\omega_{r}$ the frequency scaling the mechanical effects due to the coupling with light (when these are due to the phase or intensity gradient of the light wave, $\omega_{r}$ is the recoil frequency; Here, $\omega_{r} \sim(\Lambda / \hbar)^{2} / \nu$, with $\Lambda$ the strength of the strain coupling). In this regime and for $\gamma=0$ radiation cools the vibrations to the asymptotic occupation $N_{0}$, which is given by

$$
N_{0}=\frac{A_{+}}{A_{-}-A_{+}}=\frac{A_{+}}{\tilde{\Gamma}},
$$

and whose minimum scales with $N_{0} \sim\left(\Gamma_{\min } / \nu\right)^{2}$.

In a solid-state environment, where the heating rate due to the coupling with the external reservoir is not negligible, slowing down the cooling dynamics can be detrimental. This is visible when considering the final occupation:

$$
n_{f}=\frac{\tilde{\Gamma}}{\tilde{\Gamma}+\gamma} N_{0}+\frac{\gamma}{\tilde{\Gamma}+\gamma} N_{\mathrm{th}},
$$

where $N_{\text {th }}$ is the mean phonon occupation at the temperature of the external reservoir. Thus, maximizing the ratio $\Gamma_{\min } / \gamma$ and minimizing the ratio $\Gamma_{\min } / \nu$ is crucial and limits the parameter interval where cavity-assisted cooling can improve the efficiency.

From these considerations one can generally identify the regime where the coupling with a resonator can increase the sideband cooling efficiency. In fact, a large cavity decay rate such that $\kappa>\nu>\Gamma$ would increase the cooling rate $\tilde{\Gamma}$. Yet it can also increase the asymptotic occupation number of the mechanical mode $N_{0}$. On the other hand, a very good cavity with $\kappa<\Gamma<\nu$ can allow one to achieve smaller values of $N_{0}$, but at the price of decreasing $\tilde{\Gamma}$, so that the final occupation number of the mechanical mode $n_{f}$ becomes effectively larger.

The parameter regime to explore is quite large. However in general we expect that, in the regime where laser sideband cooling is efficient, the coupling to a resonator at linewidth $\kappa>\Gamma$ can be of help only if it substantially increases the cooling rate keeping $N_{0}<1$. The coupling to a resonator with $\kappa<\Gamma<\nu$ can help in reaching ultralow temperatures, provided thermalization can be neglected. In this article we limit our analysis by taking the optimal parameters for sideband cooling of an NV center and adding the coupling with a cavity with linewidth $\kappa \sim \Gamma$, in order to search for possible effects which cannot be foreseen drawing from these simple considerations. We refer the reader to Sec. IV where the 
choice of the parameter regime is discussed in relation to existing experimental implementations. The cooling rate, the asymptotic temperature, and the spectrum of resonance fluorescence are then determined and discussed in Sec. VI in the presence and in the absence of the coupling with the optical cavity mode. The reader who is solely interested in the resulting cooling efficiency can skip Sec. V] and jump directly to Sec. VI]

\section{THE SYSTEM}

In this Section we introduce the theoretical model which is at the basis of our study. We describe the interaction of a high- $\mathrm{Q}$ mechanical resonator mode of a phononic crystal cavity, with a quantum emitter, specifically, a NV center in diamond, and a high-finesse optical resonator mode of a photonic crystal cavity. The NV center is strain-coupled with the mechanical resonator and the electronic dipole transitions strongly couple with the photonic mode. The mechanical resonator, in turn, is optomechanically coupled to the photonic cavity. The interactions in this system are expected to be strongly enhanced by the co-localization in a single structure ensuring a perfect spatial overlap between the different degrees of freedom, which is achieved by assemblance in a monolithic diamond structure sketched in Fig. 11(a). The system is intrinsically dissipative due to radiative decay of the electronic excitations and optical cavity losses. Additionally, the mechanical resonator couples to an external thermal reservoir. We assume that it is continuously driven by a laser field, which directly couples to an electric dipole transition of the defect. In what follows we define the master equation governing the dynamics of the density matrix $\rho$, which describes the state of the composite system composed by the NV center, and the photonic and phononic resonators.

\section{A. Basic equations}

The dynamics of the hybrid system's density operator $\rho$, describing the state of the system composed by the internal degrees of freedom of the NV-center, of the optical cavity mode and of the mechanical oscillator, is governed by the master equation $\partial_{t} \rho=\mathcal{L} \rho$, where superoperator $\mathcal{L}$ is defined as $(\hbar=1)$ :

$$
\mathcal{L} \rho=-i[H, \rho]+\mathcal{L}_{\text {dis }} \rho,
$$

and which will be conveniently reported in the reference frame rotating with the laser frequency $\omega_{\mathrm{L}}$. Below we provide the detailed form of Hamiltonian $H$ and superoperator $\mathcal{L}_{\text {dis }}$.

\section{Unitary dynamics}

We first give the detailed form of the Hamiltonian $H$, which generates the unitary part of the time evolution. For convenience, we decompose it into the sum of Hermitian operators:

$$
H=H_{\text {mec }}+H_{\mathrm{I}}+\left(a+a^{\dagger}\right) V,
$$

where $a$ and $a^{\dagger}$ annihilate and create, respectively, a mechanical vibration at frequency $\nu$, while $V$ acts on the cavity and NV-center degrees of freedom and is specified later on. Operator

$$
H_{\mathrm{mec}}=\nu a^{\dagger} a
$$

is the internal energy of the mechanical resonator, while Hamiltonian $H_{\mathrm{I}}$ describes the coupled dynamics of the NV center and the optical cavity:

$$
\begin{aligned}
H_{\mathrm{I}}= & \left(\omega_{y}-\omega_{\mathrm{L}}\right)|y\rangle\left\langle y\left|+\left(\omega_{x}-\omega_{\mathrm{L}}\right)\right| x\right\rangle\langle x|+\left(\omega_{\mathrm{c}}-\omega_{\mathrm{L}}\right) c^{\dagger} c \\
& +\left[\frac{\Omega}{2}|y\rangle\langle g|+\left(g_{x}|x\rangle\left\langle g\left|+g_{y}\right| y\right\rangle\langle g|\right) c+\text { H.c. }\right] \cdot \quad \text { (9) }
\end{aligned}
$$

Here, $\omega_{x}\left(\omega_{y}\right)$ is the frequency splitting in the laboratory frame between the excited state $|x\rangle(|y\rangle)$ and the ground state $|g\rangle$; operators $c$ and $c^{\dagger}$ annihilate and create, respectively, a cavity photon at frequency $\omega_{c}$ (in the laboratory frame). The splitting between the $|x\rangle$ and $|y\rangle$ states is, for instance, due to a non-zero strain coupling, which is not related to the mechanical mode we consider. The frequencies appear shifted by $\omega_{\mathrm{L}}$ since Hamiltonian $H_{\mathrm{I}}$ is reported in the reference frame rotating at the laser frequency. The second line of Eq. (9) describes, from left to right, the external laser driving the transition $|g\rangle \rightarrow|y\rangle$ with Rabi frequency $\Omega$, while the optical mode drives the transitions $|g\rangle \rightarrow|x\rangle$ and $|g\rangle \rightarrow|y\rangle$ with vacuum Rabi frequency $g_{x}$ and $g_{y}$, respectively. We note that the laser polarization can be chosen to selectively drive one electronic transition, as we do in our model, while in general the cavity mode's polarization has a finite projection to the dipole moment of both transitions, since this depends on the preparation of the sample. Therefore, we generally assume $g_{x}, g_{y} \neq 0$ unless otherwise stated. The relevant NV center and cavity states are reported in Fig. 1(b)-(c) with the relative detunings with respect to the laser frequencies. These are defined as:

$$
\begin{aligned}
& \delta_{\mathrm{L}}=\omega_{\mathrm{L}}-\omega_{\mathrm{y}}, \\
& \Delta=\omega_{\mathrm{x}}-\omega_{\mathrm{y}}, \\
& \Delta_{\mathrm{c}}=\omega_{\mathrm{c}}-\omega_{\mathrm{y}} .
\end{aligned}
$$

Finally, operator $V$ is the sum of the strain and of the optomechanical coupling of the mechanical resonator with NV center and optical cavity, respectively. We decompose it hence into the sum $V=V_{\text {strain }}+V_{\text {om }}$, where $V_{\text {strain }}$ acts on the $\mathrm{NV}$ degrees of freedom and reads 28

$$
V_{\text {strain }}=\sum_{j=I, X, Z} \Lambda_{j} A_{j},
$$


where $\Lambda_{j}$ are the strain coupling constants and the operators $A_{j}$ are defined as:

$$
\begin{aligned}
A_{I} & =|x\rangle\langle x|+| y\rangle\langle y|, \\
A_{X} & =|x\rangle\langle y|+| y\rangle\langle x|, \\
A_{Z} & =|x\rangle\langle x|-| y\rangle\langle y| .
\end{aligned}
$$

The optomechanical coupling reads $V_{\text {om }}=-\chi c^{\dagger} c$ with $\chi$ the optomechanical coupling constant [29, 30].

\section{Dissipation}

The irreversible processes we consider in our theoretical description are: (i) the radiative decay of the NV excitations and pure dephasing of the electronic coherences, (ii) cavity losses, and (iv) the mechanical damping rate due to the coupling of the mechanical resonator with an external thermal reservoir. We model each of these phenomena by a Born-Markov process described by the corresponding superoperator, such that superoperator $\mathcal{L}_{\text {dis }}$ in Eq. (6) can be cast in the form

$$
\mathcal{L}_{\mathrm{dis}}=\mathcal{L}_{\Gamma}+\mathcal{L}_{\kappa}+\mathcal{L}_{\gamma}
$$

The individual terms read

$$
\begin{aligned}
\mathcal{L}_{\Gamma} & =\frac{\Gamma}{2} \sum_{\xi=x, y} \mathcal{D}[|g\rangle\langle\xi|]+\frac{\Gamma_{\phi}}{2} \sum_{\xi=x, y} \mathcal{D}[|\xi\rangle\langle\xi|], \\
\mathcal{L}_{\kappa} & =\frac{\kappa}{2} \mathcal{D}[c] \\
\mathcal{L}_{\gamma} & =\frac{\gamma}{2}\left(N_{\mathrm{th}}+1\right) \mathcal{D}[a]+\frac{\gamma}{2} N_{\mathrm{th}} \mathcal{D}\left[a^{\dagger}\right],
\end{aligned}
$$

where we used the definition

$$
\mathcal{D}[o] \rho=2 o \rho o^{\dagger}-o^{\dagger} o \rho-\rho o^{\dagger} o,
$$

with $o=|g\rangle\langle\xi|,| \xi\rangle\langle\xi|, c, a, a^{\dagger}$. The coefficients are the radiative decay rate $\Gamma$ of the $\mathrm{NV}$-center excited states, the dephasing rate of the electronic coherences $\Gamma_{\phi}$, cavity losses at rate $\kappa$, and the damping rate of the mechanical oscillator $\gamma$. Finally, $N_{\mathrm{th}}=\left(\exp \left(\nu / k_{\mathrm{B}} T\right)-1\right)^{-1}$ is the equilibrium phonon occupation number of the bath to which the oscillator couples, with $T$ the bath's temperature.

\section{B. Spectrum of resonance fluorescence}

In what follows we will use the master equation, Eq. (6), in order to analyze the cooling efficiency of the mechanical resonator and the spectrum of the light emitted by the NV center at the steady state of the cooling dynamics. In order to better characterize the parameter regime where cooling is efficient we choose an analytical approach, which is based on a perturbative expansion of the Lioville operator and allows us to determine the cooling regime, the corresponding rate and the asymptotic temperature. This approach is reported in the following Section.

Moreover, in the regimes of interest we determine the spectrum of the scattered light, for the purpose of identifying the relevant features in the photons which are emitted by the NV center outside of the resonator. The spectrum of resonance fluorescence is, apart from a constant proportionality factor, the Fourier transform of the auto-correlation function of the electric field [31]:

$$
\mathcal{S}(\omega) \propto \operatorname{Re} \int_{0}^{\infty} \mathrm{d} \tau \mathrm{e}^{-i \omega \tau}\left\langle E^{(-)}(\tau) E^{(+)}(0)\right\rangle_{\mathrm{st}}
$$

where $E^{(-)}(t)$ and $E^{(+)}(t)$ are the negative and positive frequency component of the electric field at time $t$ and $\langle\cdot\rangle_{\text {st }} \equiv \operatorname{Tr}\left\{\cdot \rho_{\text {st }}\right\}$ denotes the trace taken over the steady state density matrix $\rho_{\text {st }}$ which solves $\mathcal{L} \rho_{\text {st }}=0$. The intensity of the scattered field (away from the forward direction) is proportional to the source field, hence in the far-field the electric field is proportional to the sum of the operators $\mathbf{d}_{j}|g\rangle\langle j|+$ H.c., for $j=x, y$ where $\mathbf{d}_{x}$ and $\mathbf{d}_{y}$ are the dipole moments of the transitions $|g\rangle \rightarrow|x\rangle$ and $|g\rangle \rightarrow|y\rangle$, respectively (notice that $\left|\mathbf{d}_{x}\right|=\left|\mathbf{d}_{y}\right|$ ). Since the dipole moments are mutually orthogonal, the spectrum integrated over the full solid angle $4 \pi$ is the incoherent sum of the two components coming from the $|g\rangle\langle x|$ and $|g\rangle\langle y|$ operators, i.e. the interference term integrates to zero. With the help of the quantum regression theorem 32] one can cast the spectrum into the form

$$
\mathcal{S}(\omega) \propto \sum_{j=x, y} \operatorname{Re} \operatorname{Tr}\left\{|j\rangle\left\langle g\left|\left[i\left(\omega-\omega_{\mathrm{L}}\right)-\mathcal{L}\right]^{-1}\right| g\right\rangle\langle j| \rho_{\mathrm{st}}\right\} .
$$

In this work we numerically determine the spectrum for the parameter regimes of interest.

\section{PARAMETER REGIME}

In order to justify the experimental relevance of the cooling dynamics we discuss in the rest of this article, we now relate the theoretical model to existing experimental realisations and identify the parameter regime which we will consider in our analysis.

Optical resonator. A structure like the one discussed here can be found for instance in a so-called phoxonic crystal $(\mathrm{PxC})$, which co-localizes confined optical and mechanical resonator modes [9]. Photonic crystals are formed by a periodic modulation of the refractive index (in this case air holes in diamond), resulting in the formation of optical bands similar to electronic band structures in solids. A local defect like e.g. a variation of the hole diameters along the $\mathrm{PxC}$ structure perturbs the perfect periodicity and gives rise to an optical cavity mode. So far, fabrication imperfections limit experimental quality factors to $10^{4}$ at visible wavelengths suitable for the interaction with colour centers in diamond and up to $10^{5}$ in the telecom band around $1550 \mathrm{~nm}$ [10, 33 35. Nevertheless, simulations of one-dimensional photonic crystal 
cavities designed for visible light predict quality factors up to $10^{7}$ and mode volumes around 1 cubic wavelength with cavity loss rate in the range $\kappa \sim 10 \mathrm{MHz}-1 \mathrm{GHz}[9]$.

Mechanical resonator: In a $\mathrm{PxC}$ a periodic variation of the elastic modulus creates a mechanical band structure and a suitable variation of the regular pattern allows for a localized mode of the mechanical resonator. Recent experiments with structures at mechanical frequencies of $6 \mathrm{GHz}$ with optical properties suitable for telecom wavelengths show mechanical quality factors of $10^{3}$ [35. Numerical modeling shows that modes with frequencies in the range $10-20 \mathrm{GHz}$ with quality factors reaching $10^{7}$ can be achieved with an effective mass of $10^{-16} \mathrm{~kg}$ for structure dimensions matching visible wavelengths with the confined optical mode [9]. The parameters we choose are consistent with assuming mechanical frequencies of the order of $1-10 \mathrm{GHz}$ and a quality factor of the order of $10^{6}-10^{7}$, giving a damping rate $\gamma$ of few $\mathrm{kHz}$. The strain coupling constants are taken to be of the order of $1-10 \mathrm{MHz}$ [21, 36, 37. The optomechanical coupling constant $\chi$ is taken to be of the order of few $\mathrm{MHz}[9$.

$N V$ center. Figure 1(b)-(c) reports the relevant level structure of the NV center in diamond. In absence of strain coupling, the $m_{s}=0$ ground state $\left|{ }^{3} A_{20}\right\rangle$ can be selectively coupled to the excited states $\left|E_{x, y}\right\rangle$, which have zero spin angular momentum. While the ground state is much less sensitive to lattice distortion, these excited states are highly susceptible to external perturbations 2, 36, 38. Axial strain (parallel to the NV center axis, equivalent $\langle 111\rangle$ crystal direction) leads to an additional splitting between ground and excited states as well as between the $m_{s}=0$ and $m_{s}= \pm 1$ levels in the ground state. Radial strain (perpendicular to NV axis) mixes the excited state levels $E_{x}$ and $E_{y}$ and leads to a splitting of the new states $E_{x}^{*}$ and $E_{y}^{*}\left(m_{s}=+1^{*}\right.$ and $\left.m_{s}=-1^{*}\right)$. The effect of strain coupling on the excited states is several orders of magnitude larger than on the ground state and hence dominates the strain-induced modification of the NV's optical properties. Therefore, we restrict our model to the interaction between the mechanical resonator mode and the transition coupling the ground state $|g\rangle \equiv\left|{ }^{3} A_{20}\right\rangle$ to the excited states $|x\rangle \equiv\left|E_{x}\right\rangle$ and $|y\rangle \equiv\left|E_{y}\right\rangle$. For the excited states we take the radiative decay rate $\Gamma \sim 100 \mathrm{MHz}[39$, 40]. The interaction between the NV transitions and the $71 \mathrm{meV}$ lattice phonon modes [41] changes the energy of the $|x\rangle$ and $|y\rangle$ states and can thus give rise to a dephasing mechanism of the electronic coherence [3, 42. In our model we neglect the mixing between the states and consider only pure dephasing with rates of the order of $\Gamma_{\phi} \sim 100 \mathrm{MHz}$, which can be achieved in bulk diamond at temperatures lower than $10 \mathrm{~K}[28,43]$. We restrict the frequency of the mechanical resonator mode to $\nu=2 \pi \times 1 \mathrm{GHz}$ in order to avoid coupling to NV excited states other that $E_{x}$ and $E_{y}$. As the optical cavity mode should still be near resonant on the optical transition of the NV at $637 \mathrm{~nm}$ this doesn't correspond to a real structure design for the full threefold hybrid-system. However, we still model this artificial pa- rameter set in order to obtain qualitative results on the nature of the interaction.

Cooling regime: The analysis of the cooling efficiency is performed by determining the cooling rate $\tilde{\Gamma}$ and the ideal asymptotic occupation number of the mechanical mode $N_{0}$ as a function of the tunable parameters, which we take here to be the frequency splitting of the electronic excited states and the laser frequency, corresponding to changing $\delta_{\mathrm{L}}, \Delta_{\mathrm{c}}$, and $\Delta$. The analysis is performed by searching for the parameter regime where the asymptotic occupation number $N_{0}<1$ and the cooling rate $\tilde{\Gamma}$ is maximized, in order to realise regimes where the radiative cooling can overcome thermalization by the external reservoir. This constrains the range of parameters. A necessary condition for performing ground state cooling is the presence of a resonance whose linewidth $L$ is smaller than the trap frequency 44, which poses an upper bound to $L$. Moreover, the cooling rate shall exceed the thermalization rate. Since the cooling rate is proportional to the effective linewidth of the cooling transition, this condition sets a lower bound to $L$. If one performs optomechanical cooling by driving the optical resonator, then $L=\kappa$. In absence of the resonator, the mechanical oscillator can be cooled by driving the NV center transitions with a laser and $L=\Gamma$. When the NV center transitions also couple with the optical cavity, then $L$ is a linear interpolation of the cavity linewidth $\kappa$ and of the NV transition linewidth $\Gamma$, and varies between $\Gamma$ and $\kappa$ 24] (smaller linewidths could be achieved by coupling to other stable electronic transitions, which in our system are not considered [27, 45]).

In order to get a relatively small phonon occupation of the bath $N_{\text {th }}$ we take a large mechanical frequency, $\nu \sim 2 \pi \times 1 \mathrm{GHz}$, and thus for our parameter choice $\Gamma<\nu$. We then fix the cavity linewidth $\kappa \simeq \Gamma$.

\section{EFFECTIVE DYNAMICS OF THE MECHANICAL RESONATOR}

For the parameter regime we consider all characteristic frequencies characterizing the coupling of the mechanical resonator with NV center and optical cavity are much smaller than the mechanical resonator eigenfrequency $\Lambda_{I}, \Lambda_{X}, \Lambda_{Z}, \chi \bar{n}_{\mathrm{c}} \ll \nu\left(\bar{n}_{\mathrm{c}}\right.$ being the mean intracavity photon occupation number). This justifies a perturbative treatment, which allows us to eliminate the degrees of freedom of NV and optical cavity from the dynamics of the mechanical oscillator in second-order perturbation theory. By means of this procedure we derive an effective master equation for the mechanical resonator only, which allows us to determine the parameter regime where the vibrations are cooled, the corresponding cooling rate and the asymptotic vibrational state. 


\section{A. Perturbative expansion}

We derive a closed master equation for the mechanical oscillator starting from Eq. (6) and assuming that the coupling frequencies, which scale the operator $a+a^{\dagger}$, are much smaller than $\nu$. This can be summarized by the inequality $\alpha \ll \nu$, with $\alpha=\Lambda_{I}, \Lambda_{X}, \Lambda_{Z}, \chi \bar{n}_{\mathrm{c}}$ and $\bar{n}_{\mathrm{c}}$ the mean intracavity photon occupation number. We then perform perturbation theory in second order in the small parameter $\alpha / \nu$. We further assume that the incoherent dynamics of the oscillator due to the coupling with the environment is sufficiently slow that the occurrence of these processes during a scattering process can be discarded. This requires that $\gamma N_{\text {th }} \ll \alpha$, which for the parameters considered in this work is valid also at room temperature, so that we treat it in first order.

According to these considerations we split the Liouville operator as

$$
\mathcal{L}=\mathcal{L}_{0}+\mathcal{V}+\mathcal{L}_{\gamma}
$$

with $\mathcal{L}_{0}=\mathcal{L}_{\mathrm{E}}+\mathcal{L}_{\mathrm{I}}$, where $\mathcal{L}_{\mathrm{E}}$ and $\mathcal{L}_{\mathrm{I}}$ are the Liouville operators that generate the uncoupled mechanical oscillator and internal (NV center + optical cavity) dynamics, respectively, while $\mathcal{V}$ describes the coupling between mechanical and internal degrees of freedom. In detail,

$$
\begin{aligned}
\mathcal{L}_{\mathrm{E}} \rho & =-i\left[H_{\mathrm{mec}}, \rho\right], \\
\mathcal{L}_{\mathrm{I}} \rho & =-i\left[H_{\mathrm{I}}, \rho\right]+\mathcal{L}_{\Gamma} \rho+\mathcal{L}_{\kappa} \rho, \\
\mathcal{V} \rho & =-i\left[V\left(a+a^{\dagger}\right), \rho\right] .
\end{aligned}
$$

We formally eliminate the coupling between mechanical resonator and internal degrees of freedom as done for instance in Refs. 24, 46, 48. We first introduce the superoperators $\mathcal{P}_{k}$ such that

$$
\mathcal{P}_{k} \rho=\sigma_{\text {st }} \sum_{n=0}^{\infty}|n\rangle\langle n+k|\langle n|\mu| n+k\rangle,
$$

with $\mu=\operatorname{Tr}_{\mathrm{I}}\{\rho(t)\}$ the reduced density matrix, $\operatorname{Tr}_{\mathrm{I}}\{\cdot\}$ being the trace over the internal degrees of freedom, $|n\rangle$ the eigenstates of the mechanical oscillator, $k=0, \pm 1, \pm 2, \ldots$ $(k \geq-n)$ and $\sigma_{\text {st }}$ the steady state for the internal degrees of freedom: $\mathcal{L}_{\mathrm{I}} \sigma_{\text {st }}=0$. Applying $\mathcal{P}_{k}$ to the master equation (6), with the definitions of the superoperators 201-22, in a second-order perturbative expansion in parameter $\alpha / \nu$ and first order in $\gamma\left(N_{\text {th }}+1\right)$, leads to the equation

$\frac{\partial}{\partial t} \mathcal{P}_{k} \rho=\left\{i k \nu+\mathcal{P}_{k} \mathcal{V}\left(i k \nu-\mathcal{L}_{0}\right)^{-1} \mathcal{Q}_{k} \mathcal{V} \mathcal{P}_{k}\right\} \mathcal{P}_{k} \rho+\mathcal{L}_{\gamma} \mathcal{P}_{k} \rho$

with $\mathcal{Q}_{k}=1-\mathcal{P}_{k}$ and 1 is here the superoperator whose action is the identity on both sides of the density matrix. The master equation for the reduced density matrix $\mu$ is obtained after tracing out the internal degrees of freedom in Eq. 24 and reads

$$
\dot{\mu}=-i \bar{\nu}\left[a^{\dagger} a, \mu\right]+\frac{A_{-}}{2} \mathcal{D}[a] \mu+\frac{A_{+}}{2} \mathcal{D}\left[a^{\dagger}\right] \mu+\mathcal{L}_{\gamma} \mu .
$$

The rates $A_{ \pm}$are defined as

$$
\begin{gathered}
A_{ \pm}=2 \operatorname{Re} s(\mp \nu), \\
\bar{\nu}=\nu+\operatorname{Im} s(\nu)+\operatorname{Im} s(-\nu),
\end{gathered}
$$

with

$$
s(v)=\int_{0}^{\infty} d t e^{i v t}\left\langle V \exp \left(\mathcal{L}_{\mathrm{I}} t\right) V\right\rangle_{\mathrm{st}},
$$

which is the Fourier component at frequency $\nu$ of the autocorrelation function of operator $V$, where the average $\langle\cdot\rangle_{\text {st }}$ is taken in the steady state $\sigma_{\text {st }}$.

The diagonal elements of Eq. 25 give a set of rate equations for the occupation $p_{n}=\langle n|\mu| n\rangle$ of the phonon state $|n\rangle$, which are reported in Eq. (1).

\section{RESULTS}

In this section we characterize the parameter regimes in which the mechanical resonator is cooled by photon scattering process in the setup of Fig. 1 (a). We focus on the range of parameters discussed in Sec. IV. We consider laser cooling of the mechanical resonator by strain coupling with the NV center and analyse how the cooling dynamics is affected by the presence of the optical resonator and of dephasing. The results we report are compared to the predictions in absence of the optical resonator and for vanishing dephasing. This latter case has been extensively discussed in Ref. 21 and we refer the interested reader to it for a detailed discussion of the predicted dynamics in that specific limit.

\section{A. Cavity-assisted cooling}

We now analyse how laser cooling dynamics of the mechanical resonator by strain coupling with the NV center is affected by the presence of the optical resonator. In order to better understand the role of the resonator, we first discard thermal effects and dephasing (setting $\left.\gamma=\Gamma_{\phi}=0\right)$.

Figure 2 displays the cooling rate $\tilde{\Gamma}$ and the mean vibrational number at the asymptotics $N_{0}$ as a function of $\delta_{\mathrm{L}}$ and $\Delta$ in absence (left panels) and in presence of the optical cavity (right panel). Both plots show that the cooling rate is maximum, and the final occupation minimum, along the lines $\delta_{\mathrm{L}}=-\nu$ and $\delta_{\mathrm{L}}=\Delta-\nu$. In the first case cooling is achieved by setting the laser frequency to the value $\omega_{\mathrm{L}}=\omega_{y}-\nu$, hence resonantly driving the transition $|g, n\rangle \rightarrow|y, n-1\rangle$ (red sideband). In the second case the laser frequency is $\omega_{\mathrm{L}}=\omega_{x}-\nu$, so that the transition $|g, n\rangle \rightarrow|x, n-1\rangle$ is resonantly driven by an effective process, which combines the laser and the strain coupling. For most values of the detuning $\Delta$ the excitation of the intermediate state $|y\rangle$ is virtual, except for $\Delta=\omega_{x}-\omega_{y}=\nu$. This latter case corresponds to the vertical line visible in both figures, where cooling results 


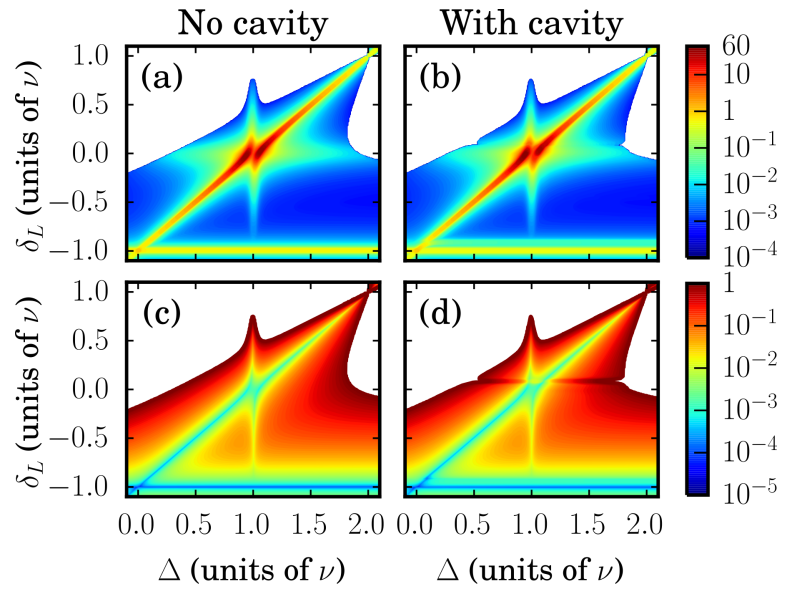

FIG. 2. (Color online) Predictions on the cooling efficiency extracted from the rate equation, Eq. (1), for laser cooling of the mechanical resonator by driving the NV center with a laser (left panel) and by additionally coupling the dipole transitions to an optical cavity (right panel). (a) and (b) show the cooling rate $\tilde{\Gamma}$, Eq. (3) in units of $\Lambda^{2} / \nu$, (c) and (d) the asymptotic occupation $N_{0}$ of the vibrational mode, according to Eq. (4), as a function of the excited level splitting $\Delta$ and the laser detuning $\delta_{\mathrm{L}}$ (in units of $\nu$ ). The white region are heating regions $(\tilde{\Gamma}<0)$ or where $N_{0}>1$. The parameters for the left panel are $\Omega=0.1 \nu, \Gamma=1.6 \times 10^{-2} \nu, \Gamma_{\phi}=0$, $\Lambda_{I}=0, \Lambda_{X}=\Lambda_{Z}=\chi=\Lambda=0.1 \Gamma$ and $g_{x}=g_{y}=0$. In the right panel we take the same parameters except for $g_{x}=g_{y}=\kappa=\Gamma$. The cavity frequency is fixed to the value $\Delta_{\mathrm{c}}=8.5 \times 10^{-2} \nu($ see text $)$.

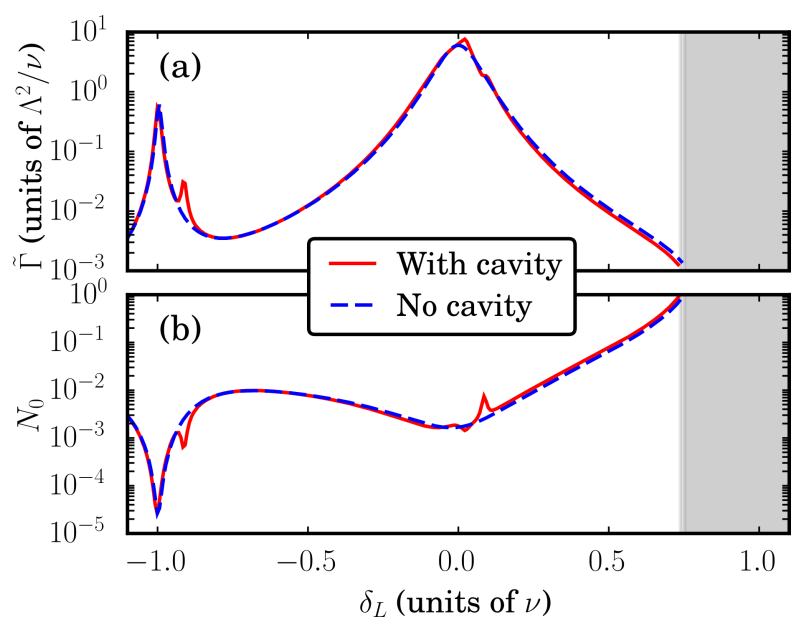

FIG. 3. (Color online) (a) Cooling rate $\tilde{\Gamma}$ and (b) asymptotic occupation $N_{0}$ of the vibrational mode as a function of $\delta_{\mathrm{L}}$ for the same parameters as in Fig. 2 and $\Delta=\nu$. The dashed (solid) line corresponds to the predictions in absence (presence) of the coupling to the cavity. The shaded region indicates the regime where the resonator is heated by the radiative processes $(\tilde{\Gamma}<0)$ or where $N_{0}>1$.

to be efficient. These properties have been identified and discussed in Ref. [21] and do not depend on the coupling with the resonator. The curves in Fig. 3 show the cooling rate and the minimum phonon occupation as a function of $\delta_{\mathrm{L}}$ after fixing the detuning $\Delta=\nu$. Some (relatively small) differences are visible close to the values $\delta_{\mathrm{L}}=0$ and $\delta_{\mathrm{L}}=-\nu$, which are due to the level splitting induced by the strong coupling with the resonator: for this choice of $\Delta_{c}$, in fact, the cavity drives almost resonantly the transition $|g\rangle \rightarrow|y\rangle$.

We have tested that the value of the detuning $\Delta_{c}$, and thus of the cavity frequency, in Figs. 2 and 3 , leads to the best results by comparing cooling rate and final temperature for different values of $\Delta_{c}$. The results of this analysis are summarized in Fig. 4, which displays (a) the maximum cooling rate (maximized by varying $\Delta$ and $\delta_{\mathrm{L}}$ by keeping $\Delta_{c}$ fixed). The mean phonon occupation in (b) and the mean intracavity photon number in (c) are reported for the corresponding values of $\Delta$ and $\delta_{\mathrm{L}}$, at which $\tilde{\Gamma}$ is maximum. These plots show that maximal cooling rates are found for $\Delta_{c} \simeq 0$. We verified that the curves do not differ substantially if instead we search for $\Delta_{c}$ by minimizing the mean phonon number. Therefore, the contour plots in Fig. 2(b) and (d) show the optimal cooling rate and temperature in presence of the resonator. On the basis of the comparison with the plots on the left panels, we can thus conclude that the coupling with the cavity does not substantially improve the cooling efficiency for the chosen parameter regime.

We now analyse how the spectrum of resonance fluorescence is modified by the coupling with the resonator. We focus on the light emitted once the system has reached the stationary state. Figure 5 displays the resonance fluorescence spectrum in absence and in presence of the optical cavity for the parameters of Fig. 3 with $\delta_{\mathrm{L}}=0$. To better understand how the cavity modifies the dynamics, we first discuss the spectrum in absence of the cavity. In this case we observe the three broad resonances around $\omega=\omega_{\mathrm{L}}$. These are due to inelastic processes in which the motion is not involved and can be interpreted as a Mollow-type triplet [49. We further observe the narrow resonances at $\omega=\omega_{\mathrm{L}} \mp \nu$, which are the red and the blue motional sidebands of the elastic peak. Subplots 5(b) and 5(c) report the details of the sidebands of the elastic peak. These spectral components correspond to the photons emitted in the processes where a phonon is created $\left(\omega_{\mathrm{L}}-\nu\right)$ or destroyed $\left(\omega_{\mathrm{L}}+\nu\right)$ in the mechanical resonator. The motional sideband has a width of the order of $\propto \Lambda_{X}^{2}$, and appears on a broader background with linewidth $\approx \Gamma$. Our analysis shows that this structure is due to the fact that mechanical effects are dominated by the strain coupling $A_{X}$, which mixes the two excited states. For our parameter choice, where $\Delta=\nu$, this coupling is weak but resonant so that the effect of the strain coupling is particularly enhanced. Figure [5(d)-(f) displays corresponding spectra of resonance fluorescence in presence of the cavity. The significantly different features are due to the modified dressed state structure because of the strong coupling between cavity and NV center, while for both cases the cooling (heating) processes are dominated by emission along the transition $|x\rangle \rightarrow|g\rangle$ 

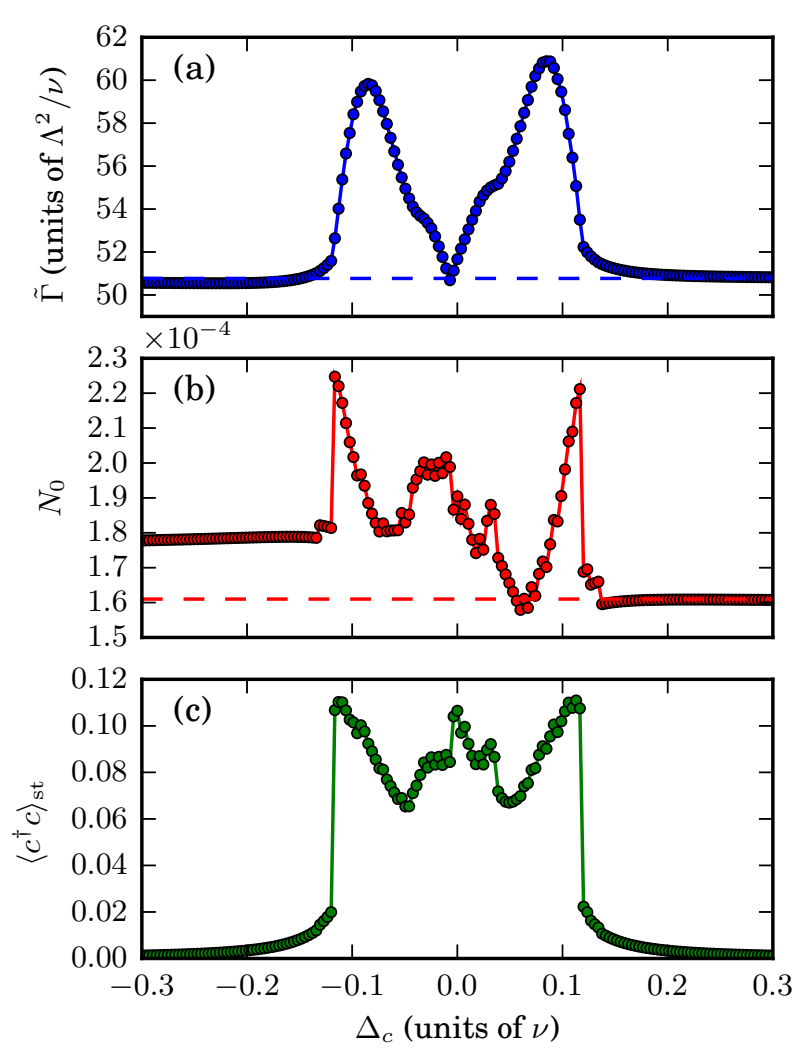

FIG. 4. (Color online) (a) Maximum cooling rate $\tilde{\Gamma}_{\max }$ in presence of the resonator as a function of $\Delta_{c}$. The value $\tilde{\Gamma}_{\max }$ has been calculated by varying $\delta_{\mathrm{L}}$ and $\Delta$ and keeping $\Delta_{\mathrm{c}}$ fixed. Subplot (b) displays the corresponding value of $N_{0}$ and (c) the mean intracavity photon number. The parameters are: $\Omega=0.1 \nu, \Gamma_{\phi}=0, \Gamma=\kappa=g_{x}=g_{y}=1.6 \times 10^{-2} \nu$, $\Lambda_{I}=0, \Lambda_{X}=\Lambda_{Z}=\chi=\Lambda=0.1 \Gamma$. The dashed lines in (a) and (b) indicate the maximum cooling rate and corresponding value of $N_{0}$ in absence of the optical resonator. In the latter case $\tilde{\Gamma}_{\max }$ is maximum for $\Delta \approx 0.93 \nu$ and $\delta_{\mathrm{L}} \approx-3.5 \times 10^{-2} \nu$.

\section{$(|y\rangle \rightarrow|g\rangle)$}

The summary of this analysis is that the effect of the optical resonator on the cooling dynamics can consist in a very small improvement of the cooling efficiency. This result, which seems to contrast with previous investigations where the effect of the cavity on the cooling efficiency was relevant [24, 27, can be understood when considering that (i) the loss rate of the resonator and the radiative decay rate of the electronic excitations have been chosen to be of the same order of magnitude, and (ii) the cooperativity $C=g^{2} / \kappa \Gamma \sim 1$, so that the level splitting induced by the coupling with the resonator is of the order of the loss rate $\kappa$. Because of (i) the coupling with the resonator gives rise to an effective level structure where the linewidths of all excited levels is of the same order of magnitude. Since for sideband cooling the linewidth determines both the cooling rate as well as the final temperature, the improvement of the cooling efficiency by coupling this level structure to a resonator is incremental. Because of (ii), the level splitting induced by the coupling with the cavity does not exceed the linewidth of the resonances, so that the regime of optimal detunings is essentially the same as without the cavity.

\section{B. Dephasing-assisted cooling}

We now analyse the effect of other noise sources on the cooling efficiency, and consider in particular dephasing, which is an important source of loss of coherence in solid-state systems. We here discard the coupling with the optical resonator and calculate the cooling efficiency when $\Gamma_{\phi} \neq 0$. Figure 6 compares the cooling rate and final occupation for $\Gamma_{\phi}=0$ (left panel) and $\Gamma_{\phi}=\Gamma$ (right panel). We observe that pure dephasing decreases the cooling efficiency when cooling is achieved by tuning the laser to the red sideband of the dressed states. Nevertheless, the cooling region is larger and the dependence on the exact values of the experimental parameters is less pronounced. Moreover, the cooling performance is enhanced in most parts of parameter landscape. Figures 7(a) and (b) compare the cuts along the line $\Delta=\nu$ : one clearly sees that the case of $\Gamma_{\phi}=\Gamma$ outperforms the case when $\Gamma_{\phi}=0$. This occurs over almost the full range of $\delta_{\mathrm{L}}$ in terms of both cooling rate and minimal phonon number. We have checked that the value $\Gamma_{\phi}=\Gamma$ is close to the optimal dephasing rate. We also found the range of values in which the dephasing has a beneficial effect on the cooling spans till several $\Gamma$ (see dotted line, which shows the predictions for $\left.\Gamma_{\phi}=10 \Gamma\right)$.

The effect of dephasing is also visible in the spectrum of resonance fluorescence. We observe in Fig. 8 for $\delta_{\mathrm{L}}=0 \mathrm{a}$ broadening of the background at the motional sidebands, which now scale with $\approx \Gamma+\Gamma_{\phi}$. This linewidth is indeed the cooling rate, which results to be enhanced by the presence of pure dephasing.

We understand this behaviour since pure dephasing increases the width of the excited states $|x\rangle$ and $|y\rangle$ without increasing their decay rate. Thus it increases the excitation probability. Since this cooling scheme is optimal when population is transferred to the excited state, then pure dephasing leads to larger transition rates, and thus larger cooling rate. This reasoning works within a certain parameter interval: dephasing rates exceeding the Rabi frequency, in fact, tend to suppress population transfer and thus are detrimental.

The beneficial role of pure dephasing on the cooling efficiency can be best illustrated by analysing the final mean occupation for different temperatures of the bath. Figure 9 illustrates how dephasing can improve the cooling efficiency over a large parameter regime, flattening out the minimum of $n_{f}$ (Eq. (5)) as a function of the frequency of the driving laser. 

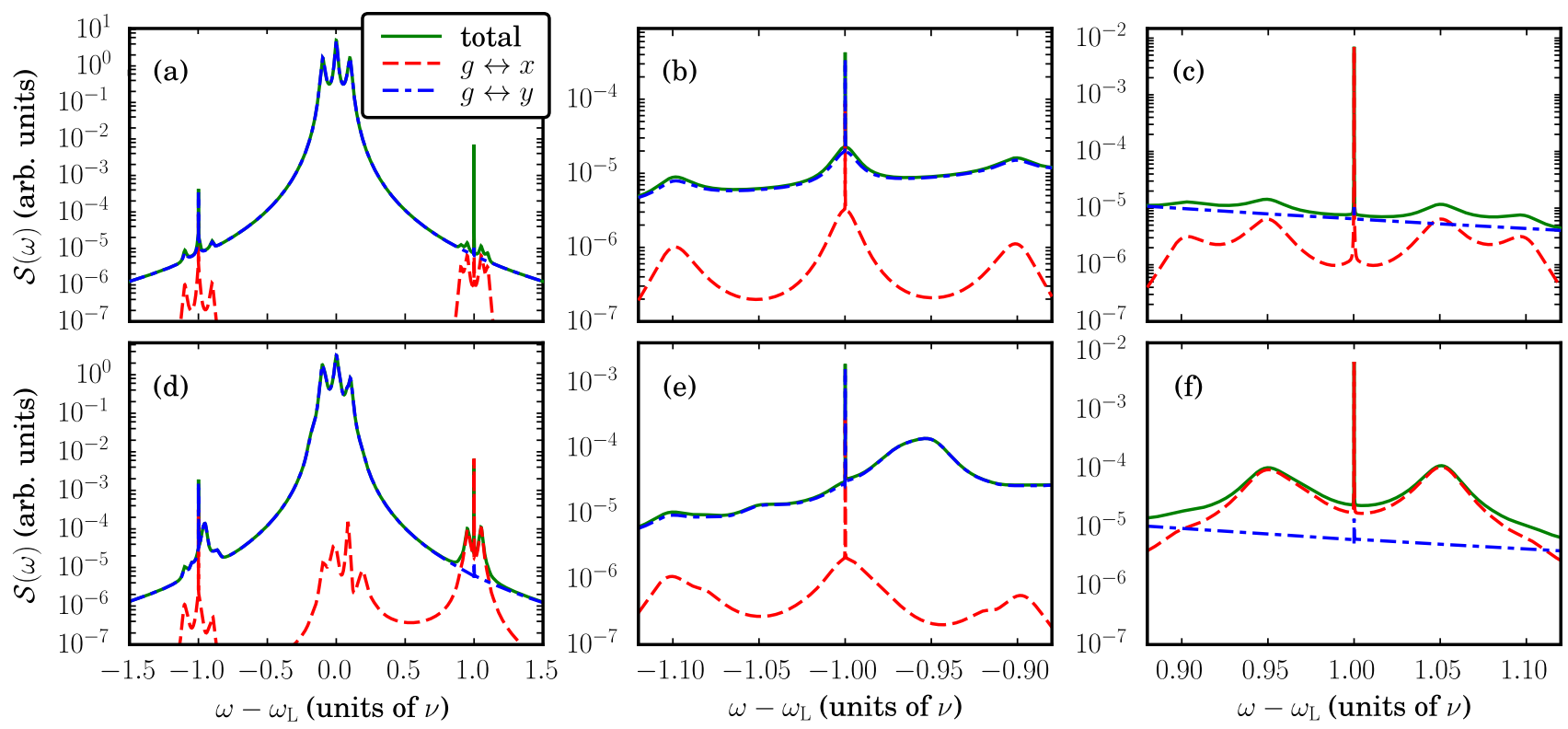

FIG. 5. (Color online) Spectrum of light emitted by the NV center at the asymptotics of the laser cooling dynamics. The upper panels correspond to the parameter regime of Fig. 2(a)(c) (no cavity), the lower panels to the parameter regime of Fig. 2(b)(d) (cavity assisted cooling). The dashed (dashed-dotted) line correspond to the emission from the transition $g \leftrightarrow x(g \leftrightarrow y)$, the solid line correspond to the sum of these two contributions. Here, we took $\Delta=\nu$ and $\delta_{\mathrm{L}}=0$. Panels (b), (c), (e) and (f) show the details of the sidebands.

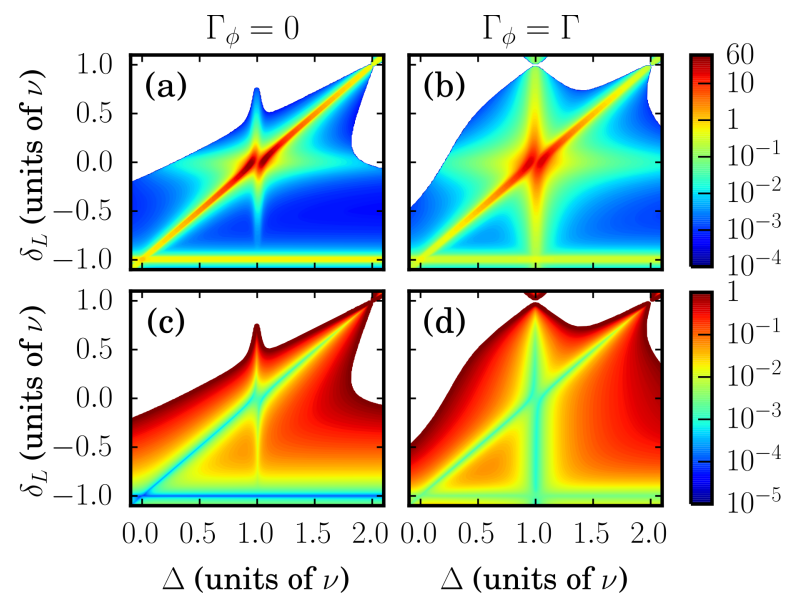

FIG. 6. (Color online) Predictions on the cooling efficiency extracted from the rate equation, Eq. (1), for laser cooling of the mechanical resonator by driving the NV center with a laser in absence (left panel) and in presence of pure dephasing (right panel). (a) and (b) show the cooling rate $\tilde{\Gamma}$, Eq. (3) in units of $\Lambda^{2} / \nu$, (c) and (d) the asymptotic occupation $N_{0}$ of the vibrational mode, according to Eq. (4), as a function of the excited level splitting $\Delta$ and the laser detuning $\delta_{\mathrm{L}}$ (in units of $\nu)$. The white area are heating regions $(\tilde{\Gamma}<0)$ or where $N_{0}>1$. The parameters are $\Omega=0.1 \nu, \Gamma=1.6 \times 10^{-2} \nu$, $\Lambda_{I}=0, \Lambda_{X}=\Lambda_{Z}=\chi=\Lambda=0.1 \Gamma$, and (left panel) $\Gamma_{\phi}=0$, (right panel) $\Gamma_{\phi}=\Gamma$.

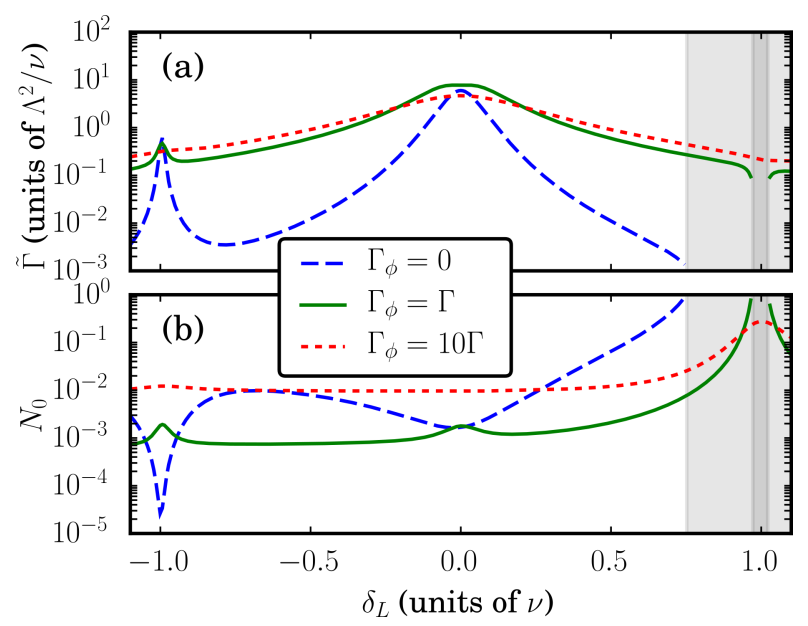

FIG. 7. (Color online) (a) Cooling rate $\tilde{\Gamma}$ and (b) asymptotic occupation $N_{0}$ of the vibrational mode as a function of $\delta_{\mathrm{L}}$ for the same parameters as in Fig. 6 and $\Delta=\nu$. The dashed line corresponds to the predictions in absence of dephasing. The solid (dotted) line corresponds to the predictions when the dephasing rate is $\Gamma_{\phi}=\Gamma\left(\Gamma_{\phi}=10 \Gamma\right)$. The shaded region indicates the regime where the resonator is heated by the radiative processes $(\tilde{\Gamma}<0)$ or where $N_{0}>1$.

\section{DISCUSSION AND CONCLUSIONS}

We have analysed the cooling efficiency of a mechanical resonator which is laser cooled by the strain coupling with a NV center. The cooling dynamics is essentially due to the strain coupling with the NV center and the 

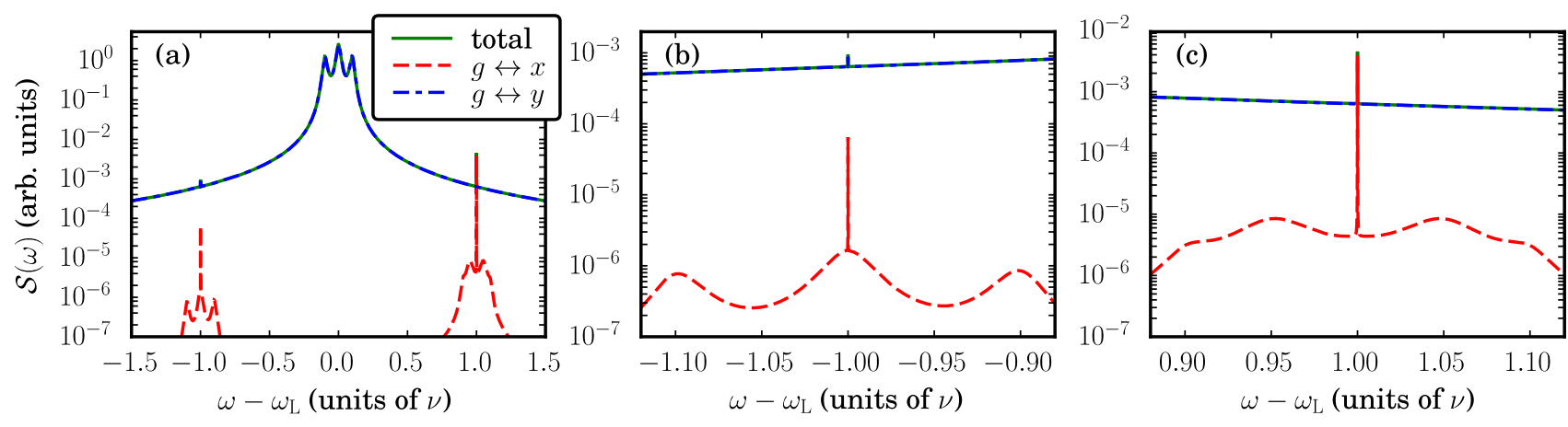

FIG. 8. (Color online) Spectrum of light emitted by the NV center at the asymptotics of the laser cooling dynamics. The parameters are the same as in Fig. 6(b)(d) (dephasing assisted cooling with $\Gamma_{\phi}=\Gamma$ ). The dashed (dashed-dotted) line correspond to the emission from the transition $g \leftrightarrow x(g \leftrightarrow y)$, the solid line correspond to the sum of these two contributions. Here, we took $\Delta=\nu$ and $\delta_{\mathrm{L}}=0$. Panels (b) and (c) show the details of the sidebands.

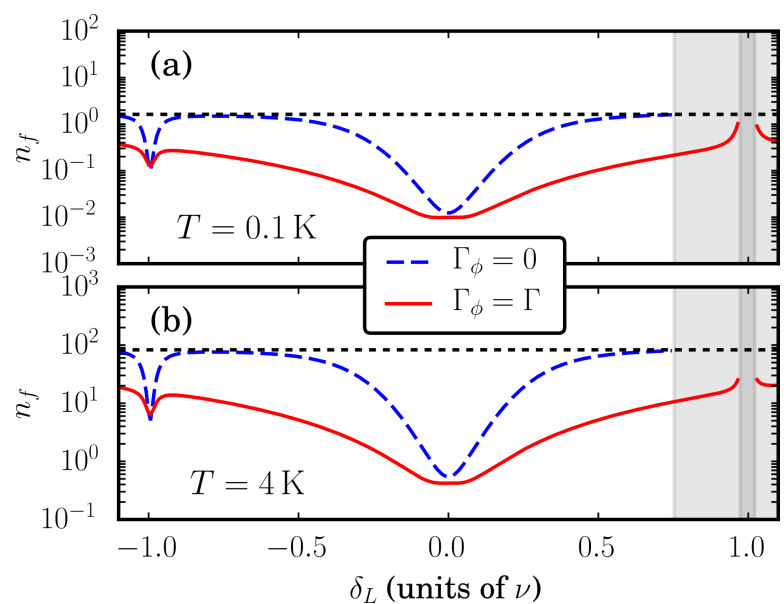

FIG. 9. (Color online) Final phonon number of the mechanical resonator with $\nu=2 \pi \times 1 \mathrm{GHz}$ and a quality factor $Q=\nu / \gamma=10^{7}$, Eq. $(5)$, for (a) $T=0.1 \mathrm{~K}\left(N_{\mathrm{th}} \approx 1.6\right)$ and (b) $T=4 \mathrm{~K}\left(N_{\mathrm{th}} \approx 83\right)$, for the same parameter regime of Fig. 7(b). The dashed (solid) line corresponds to the predictions in absence (presence) of pure dephasing. The black dotted lines correspond to $n_{f}=N_{\text {th }}$. The shaded region indicates the regime where the resonator is heated by the radiative processes.

parameter regime is such that the resolved-sideband cooling can be performed by driving the NV center electronic resonances. In this regime we have analysed the effect of the coupling to an optical resonator, and found that it does only incrementally improve the cooling efficiency. We have further shown that pure dephasing can make the cooling dynamics more robust against parameter fluctuations, without affecting the overall efficiency, as long as the dephasing rate does not exceed the driving strength of the laser.

In our analysis the optomechanical coupling was a small effect. It can be increased in configurations where the cavity is driven: in this case the optomechanical coupling would cool the resonator according to the dynamics explored in Refs. [50, 51]. Another interesting possibility is to drive both optical cavity and NV center for large cooperativity: In this situation phonon excitation or absorption can be realised by means of two excitation paths, that can interfere. This interference depends on the relative phase between the lasers and could be a control parameter for realising multi-wave mixing.

\section{ACKNOWLEDGMENTS}

We thank Peter Rabl, Stefan Schütz, and Christoph Becher for useful discussions and helpful comments. This work was partially financially supported by the DFG ("Optomechanical cavity Quantum Electrodynamics with colour centers in diamond"), the German Ministry of Education and Research (BMBF, "Q.com"), the DFG Forschergruppe FOR 1493, and the GradUS program of Saarland University.
[1] T. Schröder, S. L. Mouradian, J. Zheng, M. E. Trusheim, M. Walsh, E. H. Chen, L. Li, I. Bayn, and D. Englund, J. Opt. Soc. Am. B 33, B65 (2016).

[2] M. W. Doherty, N. B. Manson, P. Delaney, F. Jelezko, J. Wrachtrup, and L. C. Hollenberg, Phys. Rep. 528, 1 (2013).

[3] R. Albrecht, A. Bommer, C. Deutsch, J. Reichel, and C.
Becher, Phys. Rev. Lett. 110, 243602 (2013).

[4] R. Brouri, A. Beveratos, J. P. Poizat, and P. Grangier, Opt. Lett. 25, 1294 (2000)

[5] C. Kurtsiefer, S. Mayer, P. Zarda, and H. Weinfurter, Phys. Rev. Lett. 85, 290 (2000).

[6] J. Teissier, A. Barfuss, P. Appel, E. Neu, and P. Maletinsky, Phys. Rev. Lett. 113, 020503 (2014). 
[7] P. Ovartchaiyapong, K. W. Lee, B. A. Myers , and A. C. Bleszynski Jayich, Nat. Commun. 5, 4429 (2014).

[8] K. W. Lee, D. Lee, P. Ovartchaiyapong, J. Minguzzi, J. R. Maze, and A. C. Bleszynski Jayich, arXiv:1603.07680 (2016).

[9] L. Kipfstuhl, F. Guldner, J. Riedrich-Möller and C. Becher, Opt. Expr. 22, 12410 (2014).

[10] L. Li, T. Schröder, E. H. Chen, M. Walsh, I. Bayn, J. Goldstein, O. Gaathon, M. E. Trusheim, M. Lu, J. Mower, M. Cotlet, M. L. Markham, D. J. Twitchen and D. Englund, Nat. Commun. 6, (2015).

[11] E. R. MacQuarrie, T. A. Gosavi, N. R. Jungwirth, S. A. Bhave, and G. D. Fuchs, Phys. Rev. Lett. 111, 227602 (2013).

[12] E. R. MacQuarrie, T. A. Gosavi, A. M. Moehle, N. R. Jungwirth, S. A. Bhave, and G. D. Fuchs, Optica 2, 233 (2015)

[13] M. Aspelmeyer, T. J. Kippenberg, and F. Marquardt, Rev. Mod. Phys. 86, 1391 (2014).

[14] J. Wrachtrup and F. Jelezko, J. Phys. 18, S807, (2006).

[15] L. Childress and R. Hanson, MRS Bull. 38, 134 (2013).

[16] K. Nemoto, M. Trupke, S. J. Devitt, A. M. Stephens, B. Scharfenberger,K. Buczak, T. Nöbauer, M. S. Everitt, J. Schmiedmayer, and W. J. Munro, Phys. Rev. X 4, 031022 (2014).

[17] J. Wrachtrup, F. Jelezko, B. Grotz, and L. McGuinness, MRS Bull. 38, 02, 149 (2013).

[18] S. Hong, M. S. Grinolds, L. M. Pham, D. Le Sage, L. Luan, R. L. Walsworth, and A. Yacoby, MRS Bull. 38, 02, 155 (2013).

[19] R. Schirhagl, K. Chang, M. Loretz, and C. L. Degen, Annu. Rev. Phys. Chem. 65, 83 (2014).

[20] L. Rondin, J. P. Tetienne, T. Hingant, J. F. Roch, P. Maletinsky, and V. Jacques, Rep. Prog. Phys. 77, 056503 (2014).

[21] K. V. Kepesidis, S. D. Bennett, S. Portolan, M. D. Lukin, and P. Rabl, Phys. Rev. B 88, 064105 (2013).

[22] J. I. Cirac, M. Lewenstein, and P. Zoller, Phys. Rev. A 51, 1650 (1995).

[23] V. Vuletic, H. W. Chan, and A. T. Black, Phys. Rev. A 64, 033405 (2001).

[24] S. Zippilli and G. Morigi, Phys. Rev. Lett. 95, 143001 (2005); Phys. Rev. A 72, 053408 (2005).

[25] S. Zippilli, G. Morigi, and W. P. Schleich, J. Mod. Opt. 54, 1595 (2007).

[26] S. Stenholm, Rev. Mod. Phys. 58, 699 (1986).

[27] M. Bienert and G. Morigi, New J. Phys. 14, 023002 (2012).

[28] K.-M. C. Fu, C. Santori, P. E. Barclay, L. J. Rogers, N. B. Manson, and R. G. Beausoleil, Phys. Rev. Lett. 103, 256404 (2009).
[29] C. K. Law, Phys. Rev. A 49, 433 (1994).

[30] C. K. Law, Phys. Rev. A 51, 2537 (1995).

[31] R. J. Glauber, Quantum Theory of Optical Coherence (Wiley-VCH, Weinheim).

[32] H. J. Carmichael, Statistical Methods in Quantum Optics 1 (Springer, Berlin).

[33] B. J. M. Hausmann, B. J. Shields, Q. Quan, Y. Chu, N. P. de Leon, R. Evans, M. J. Burek, A. S. Zibrov, M. Markham, D. J. Twitchen, H. Park, M. D. Lukin, and M. Lončar, Nano Lett. 13, 5791 (2013).

[34] M. J. Burek, Y. Chu, M. S. Liddy, P. Patel, J. Rochman, S. Meesala, W. Hong, Q. Quan, M. D. Lukin, and M. Lončar, Nat. Commun. 5, (2014).

[35] M. J. Burek, J. D. Cohen, S. M. Meenehan, T. Ruelle, S. Meesala, J. Rochman, H. A. Atikian, M. Markham, D. J. Twitchen, M. D. Lukin, O. Painter, M. Lončar, arXiv:1512.04166 (2015).

[36] M. W. Doherty, N. B. Manson, P. Delaney, and L. C. L. Hollenberg, New J. Phys. 13, 025019 (2011).

[37] P. Tamarat, T. Gaebel, J. R. Rabeau, M. Khan, A. D. Greentree, H. Wilson, L. C. L. Hollenberg, S. Prawer, P. Hemmer, F. Jelezko, and J. Wrachtrup, Phys. Rev. Lett. 97, 083002 (2006).

[38] J. R. Maze, A. Gali, E. Togan, Y. Chu, A. Trifonov, E. Kaxiras, and M. D. Lukin, New J. Phys. 13, 025025 (2011).

[39] A. Sipahigil, M. L. Goldman, E. Togan, Y. Chu, M. Markham, D. J. Twitchen, A. S. Zibrov, A. Kubanek, and M. D. Lukin, Phys. Rev. Lett. 108, 143601 (2012).

[40] H. Bernien, L. Childress, L. Robledo, M. Markham, D. Twitchen, and R. Hanson, Phys. Rev. Lett. 108, 043604 (2012).

[41] A. Gali, T. Simon, and J. E. Lowther, New J. Phys. 13, 025016 (2011).

[42] R. Betzholz, J. M. Torres, and M. Bienert, Phys. Rev. A 90, 063818 (2014).

[43] T. A. Abtew, Y. Y. Sun, B.-C. Shih, P. Dev, S. B. Zhang, and P. Zhang, Phys. Rev. Lett. 107, 146403 (2011).

[44] J. Eschner, G. Morigi, F. Schmidt-Kaler, and R. Blatt, J. Opt. Soc. Am. B 20, 1003 (2003).

[45] G. Morigi, J. Eschner, and C. H. Keitel, Phys. Rev. Lett. 85, 4458 (2000).

[46] J. Javanainen, M. Lindberg, and S. Stenholm, J. Opt. Soc. Am. B 1, 111 (1984).

[47] J. I. Cirac, R. Blatt, P. Zoller, and W. D. Phillips, Phys. Rev. A 46, 2668 (1992).

[48] G. Morigi, Phys. Rev. A 67, 033402 (2003).

[49] B. R. Mollow, Phys. Rev. 188, 1969 (1969).

[50] I. Wilson-Rae, N. Nooshi, J. Dobrindt, T. J. Kippenberg and W. Zwerger, New J. Phys. 10, 095007 (2008).

[51] F. Marquardt, J. P. Chen, A. A. Clerk, and S. M. Girvin, Phys. Rev. Lett. 99, 093902 (2007). 